\title{
Lifetime measurements in 166Re: Collective versus magnetic rotation
}

\section{Document Version}

Final published version

Link to publication record in Manchester Research Explorer

\section{Citation for published version (APA):}

Taylor, M. (2016). Lifetime measurements in 166Re: Collective versus magnetic rotation. Physical Review C (Nuclear Physics), 93, [034309]. http://doi.org/10.1103/PhysRevC.93.034309

\section{Published in:}

Physical Review C (Nuclear Physics)

\section{Citing this paper}

Please note that where the full-text provided on Manchester Research Explorer is the Author Accepted Manuscript or Proof version this may differ from the final Published version. If citing, it is advised that you check and use the publisher's definitive version.

\section{General rights}

Copyright and moral rights for the publications made accessible in the Research Explorer are retained by the authors and/or other copyright owners and it is a condition of accessing publications that users recognise and abide by the legal requirements associated with these rights.

\section{Takedown policy}

If you believe that this document breaches copyright please refer to the University of Manchester's Takedown Procedures [http://man.ac.uk/04Y6Bo] or contact uml.scholarlycommunications@manchester.ac.uk providing relevant details, so we can investigate your claim.

\section{OPEN ACCESS}




\title{
Lifetime measurements in ${ }^{166} \mathrm{Re}$ : Collective versus magnetic rotation
}

H. J. Li, ${ }^{1,2, *}$ B. Cederwall, ${ }^{1}$ M. Doncel, ${ }^{1}$ J. Peng, ${ }^{3}$ Q. B. Chen, ${ }^{4}$ S. Q. Zhang,${ }^{4}$ P. W. Zhao, ${ }^{4}$ J. Meng,${ }^{4,5,6}$ T. Bäck, ${ }^{1}$ U. Jakobsson, ${ }^{1,7}$ K. Auranen, ${ }^{7}$ S. Bönig, ${ }^{8}$ M. Drummond, ${ }^{9}$ T. Grahn, ${ }^{7}$ P. Greenlees, ${ }^{7}$ A. Herzáń, ${ }^{7}$ D. T. Joss, ${ }^{9}$ R. Julin, ${ }^{7}$ S. Juutinen, ${ }^{7}$ J. Konki, ${ }^{7}$ T. Kröll,${ }^{8}$ M. Leino, ${ }^{7}$ C. McPeake, ${ }^{9}$ D. O’Donnell, ${ }^{9}$ R. D. Page, ${ }^{9}$ J. Pakarinen, ${ }^{7}$ J. Partanen, ${ }^{7}$ P. Peura,,${ }^{7, \dagger}$ P. Rahkila, ${ }^{7}$ P. Ruotsalainen,,${ }^{7,7}$ M. Sandzelius, ${ }^{7}$ J. Sarén, ${ }^{7}$ B. Sayğ $1,{ }^{9,}{ }^{8}$ C. Scholey, ${ }^{7}$ J. Sorri, ${ }^{7}$ S. Stolze, ${ }^{7}$ M. J. Taylor, ${ }^{10}$ A. Thornthwaite, ${ }^{9}$ J. Uusitalo, ${ }^{7}$ and Z. G. Xiao ${ }^{2}$

${ }^{1}$ Department of Physics, KTH-Royal Institute of Technology, SE-10691 Stockholm, Sweden

${ }^{2}$ Department of Physics, Tsinghua University, Beijing 100084, People's Republic of China

${ }^{3}$ Department of Physics, Beijing Normal University, Beijing 100875, China

${ }^{4}$ State Key Laboratory of Nuclear Physics and Technology, School of Physics, Peking University, Beijing 100871, China

${ }^{5}$ School of Physics and Nuclear Energy Engineering, Beihang University, Beijing 100191, China

${ }^{6}$ Department of Physics, University of Stellenbosch, Stellenbosch, South Africa

${ }^{7}$ University of Jyvaskyla, Department of Physics, P.O. Box 35, FI-40014 University of Jyvaskyla, Finland

${ }^{8}$ Institut für Kernphysik, TU Darmstadt, D-64289 Darmstadt, Germany

${ }^{9}$ Department of Physics, Oliver Lodge Laboratory, University of Liverpool, Liverpool L69 7ZE, United Kingdom

${ }^{10}$ School of Physics and Astronomy, University of Manchester, Manchester M13 9PL, United Kingdom

(Received 29 May 2015; revised manuscript received 24 December 2015; published 8 March 2016)

\begin{abstract}
Lifetimes of excited states in the neutron-deficient odd-odd nucleus ${ }^{166} \mathrm{Re}$ have been measured for the first time using the recoil distance Doppler-shift method. The measured lifetime for the $\left(8^{-}\right)$state; $\tau=480(80) \mathrm{ps,}$ enabled an assessment of the multipolarities of the $\gamma$ rays depopulating this state. Information on electromagnetic transition strengths were deduced for the $\gamma$-ray transitions from the $\left(9^{-}\right),\left(10^{-}\right)$, and $\left(11^{-}\right)$states, and in the case of the $\left(10^{-}\right)$and $\left(11^{-}\right)$states limits on the $B(M 1)$ and $B(E 2)$ strengths were estimated. The results are compared with total Routhian surface predictions and semiclassical calculations. Tilted-axis cranking calculations based on a relativistic mean-field approach (TAC-RMF) have also been performed in order to test the possibility of magnetic rotation in the ${ }^{166} \mathrm{Re}$ nucleus. While the TAC-RMF calculations predict a quadrupole-deformed nuclear shape with similar $\beta_{2}$ deformation as obtained by using the TRS model, it was found that the experimental electromagnetic transition rates are in better agreement with a collective-rotational description.
\end{abstract}

DOI: 10.1103/PhysRevC.93.034309

\section{INTRODUCTION}

Studies of odd-odd nuclei in the $Z \approx 70, A \approx 160$ region of the nuclear chart have revealed several interesting phenomena originating from the interplay between the odd valence neutron and proton as well as with a deformation soft core. Most of these neutron-deficient nuclei are predicted by theory to be deformed in the ground state, typically with a near-prolate shape at $\beta_{2} \approx 0.2$ [1]. Even though a large set of rotational-like band structures built on different quasiparticle configurations have been experimentally established in the region, it is important to extend our knowledge of excited states in oddodd deformed nuclei to the most neutron-deficient species in order to probe new combinations of neutron and proton configurations and the residual interactions between them. The energy splitting between the two signature partners of strongly coupled rotational bands and the electromagnetic transition strengths between them can provide important clues

\footnotetext{
*hongjiel@kth.se

${ }^{\dagger}$ Current address: University of Helsinki and Helsinki Institute of Physics, P.O. Box 64, FI-00014, Helsinki, Finland.

${ }^{\ddagger}$ Current address: TRIUMF, 4004 Wesbrook Mall, Vancouver, British Columbia, V6T 2A3, Canada.

${ }^{\S}$ Current address: Department of Physics, Faculty of Science, Ege University, 35100, Bornova, Izmir, Turkey.
}

to the nature of such interactions. In particular, inversion of the energies in the rotational frame of signature partner bands $[2,3]$ is a striking phenomenon of great interest. Low-spin signature inversion has been observed in the yrast bands of some odd-odd $A \approx 160$ nuclides [4-8] and band (2) in ${ }^{166} \operatorname{Re}$ [9]. The signature-inversion effect, implying that the normally energetically favored signature becomes unfavored, has been addressed by using different theoretical approaches [10-12] but still has no consistent theoretical explanation.

The relative richness in available data on excited-state energies is in contrast to the limited corresponding knowledge of transition probabilities. Such information, although more difficult to obtain experimentally, is crucial for testing theoretical models in a decisive way.

In this work we report on the first measurement of excitedstate lifetimes in the neutron-deficient nuclide ${ }^{166} \mathrm{Re}$, situated 19 neutrons away from the lightest stable rhenium isotope. This is one of few nuclei in this highly-neutron-deficient region where such information is available and the only reported lifetime measurement of an odd-odd nucleus in this region to date. The experimentally deduced electromagnetic transition strengths for the low-lying states in the yrast rotational band structure are compared with results from total Routhian surface, semiclassical model, and tilted axis cranking model calculations based on a relativistic mean-field approach. 


\section{EXPERIMENTAL METHODS AND RESULTS}

Excited states in ${ }^{166} \mathrm{Re}$ were populated with the ${ }^{92} \mathrm{Mo}\left({ }^{78} \mathrm{Kr}, 3 p 1 n\right){ }^{166} \mathrm{Re}$ fusion-evaporation reaction. The experiment was performed at the Accelerator Laboratory of the University of Jyväskylä, Finland. The ${ }^{78} \mathrm{Kr}$ beam, accelerated by the K-130 cyclotron to $380 \mathrm{MeV}$, was allowed to impinge on a $0.6-\mathrm{mg} / \mathrm{cm}^{2}$-thick ${ }^{92}$ Mo target mounted inside the differential plunger for unbound nuclear states (DPUNS) device [13]. The velocity of the recoiling fusion-evaporation residues, $v / c$, was $4.4 \%$ and $3.4 \%$ before and after a $1-\mathrm{mg} / \mathrm{cm}^{2}$-thick $\mathrm{Mg}$ degrader foil, respectively.

The prompt $\gamma$ rays were detected by the JUROGAM II germanium detector array, which consists of 15 EUROGAM Phase-I [14] and GASP-type [15] single-crystal germanium detectors and 24 EUROBALL clover germanium detectors [16]. Five of the single-crystal detectors were placed at $157.6^{\circ}$ relative to the beam direction, while 10 detectors were placed at $133.6^{\circ}$. Twelve clover detectors were located at $104.5^{\circ}$ and 12 at $75.5^{\circ}$ relative to the beam direction. The fusion-evaporation products were separated from beam particles and fission products by employing the recoil ion transport unit RITU [17-19]. The fusion reaction products were detected by two double-sided silicon strip detectors (DSSSDs) at the focal plane of RITU, forming part of the GREAT spectrometer [20]. The data were recorded with the triggerless total data readout (TDR) acquisition system [21].

In order to cover a lifetime range from a few to a few hundred picoseconds; the expected range of the lifetimes of interest, nine different target-to-degrader distances were measured: 7.6 (2), 96.4 (3), 192.3 (4), 485.2 (40), 984.1 (40), $2000(6), 3000(9), 5000$ (15), and $8000(24) \mu \mathrm{m}$. For a detailed description of the DPUNS device we refer to Ref. [13].

The recoil-gated prompt $\gamma$-ray-coincidence data were sorted into nine matrices, one for each target-to-degrader distance, by using the GRAIN [22] software package. The matrices were obtained by sorting the $\gamma$-ray energies detected in any of the clover detectors on one axis and the $\gamma$-ray energies detected in any of the ten $133.6^{\circ}$ Phase-I detectors on the other axis. The $\gamma$-ray energy coincidence matrices were analyzed using the RADWARE analysis package [23]. A Doppler correction with $v / c=4.4 \%$ (i.e., corresponding to the average nuclear velocity before the degrader foil) was applied. Excited-state lifetimes were deduced by means of the recoil distance Doppler shift (RDDS) method [24-26]. The data analysis followed the principles of the differential decay curve method (DDCM) [27,28]. Within this method, the lifetime for the excited state at each target-to-degrader distance, $x$, can be evaluated by using the expression

$$
\tau(x)=\frac{\left\{C_{0}^{\infty}, A_{t}^{\infty}\right\}-\left\{C_{0}^{\infty}, B_{t}^{\infty}\right\} \frac{\left\{C_{0}^{\infty}, A_{0}^{\infty}\right\}}{\left\{C_{0}^{\infty}, B_{0}^{\infty}\right\}}}{\frac{d}{d x}\left\{C_{0}^{\infty}, A_{0}^{t}\right\}} \frac{1}{\langle v\rangle} .
$$

Here we suppose a cascade of $\gamma$-ray transitions $l_{1} \stackrel{C_{\gamma}}{\longrightarrow} l_{2} \stackrel{B_{\gamma}}{\longrightarrow}$ $l_{3} \stackrel{A_{\gamma}}{\rightarrow} l_{4}$, where $l_{i}$ represents different levels and $l_{3}$ is the level for which the lifetime $\tau$ is evaluated. $B_{\gamma}$ is a transition directly feeding $l_{3}$, while $C_{\gamma}$ represents a transition directly feeding level $l_{2}$ in the cascade and is used to produce
(1)

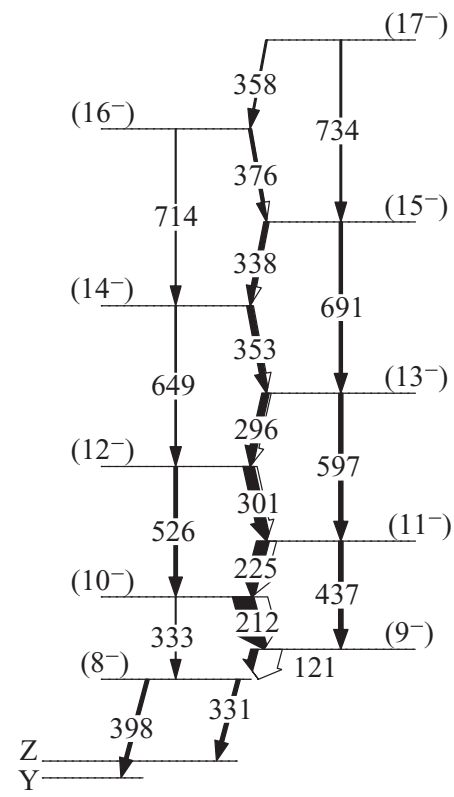

FIG. 1. Part of the level scheme of ${ }^{166}$ Re taken from our previous work [9]. Energies are in keV. The labels $\mathrm{Y}$ and $\mathrm{Z}$ indicate the states populated by the 398 and $331 \mathrm{keV} \gamma$-ray transitions, respectively.

a coincidence-gated spectrum for each target-to-degrader distance of interest. The expressions $\left\{C_{0}^{\infty}, A_{0}^{t}\right\},\left\{C_{0}^{\infty}, A_{t}^{\infty}\right\}$, and $\left\{C_{0}^{\infty}, A_{0}^{\infty}\right\}$ correspond to the fully-Doppler-shifted component $I s_{A}$, the degraded component $I d_{A}$, and both components $I s_{A}+I d_{A}$ of the $\gamma$-ray transitions of interest in these spectra, respectively. The coincidence gates were set to include both the fully shifted and degraded components of transition $C_{\gamma}$. $\left\{C_{0}^{\infty}, B_{t}^{\infty}\right\}$ and $\left\{C_{0}^{\infty}, B_{0}^{\infty}\right\}$ represent the Doppler-degraded component $I d_{B}$ and the full intensity of the peaks $I s_{B}+I d_{B}$ of the feeding $\gamma$-ray transitions, respectively, with gates including both the fully shifted and degraded components of transition $C_{\gamma}$. The observed coincidence intensities were corrected for both internal conversion and the relative coincidence efficiencies. $\langle v\rangle$ is the average velocity of the recoiling nuclei. The APATHIE [29] software was used to fit the variation of $I s_{A}$ with target-to-degrader distance. The fully-Doppler-shifted and degraded components were fit for both the feeding and depopulating transitions, with gates set on the clover detectors. By using Eq. (1), a lifetime value can be obtained for each target-to-degrader distance, while the final lifetime value is obtained from the weighted average within the sensitive region [13].

Lifetime information could in this way be obtained for four excited states in ${ }^{166} \mathrm{Re}$ : the $\left(8^{-}\right),\left(9^{-}\right),\left(10^{-}\right)$, and $\left(11^{-}\right)$ states in the yrast ban; see the partial level scheme in Fig. 1. It is noteworthy that there is significant unobserved intensity depopulating the $\left(8^{-}\right)$state. The reason could be a strong fragmentation of the decay into the low-lying single-particle states of this odd-odd nucleus or an unobserved, strongly converted $M 1$ transition to a possible lower $\left(7^{-}\right)$state in the band. However, the deduced lifetime of the $\left(8^{-}\right)$state is independent of such unobserved transitions. Both the 331 and $398 \mathrm{keV}$ depopulating $\gamma$-ray transitions have been analyzed for 


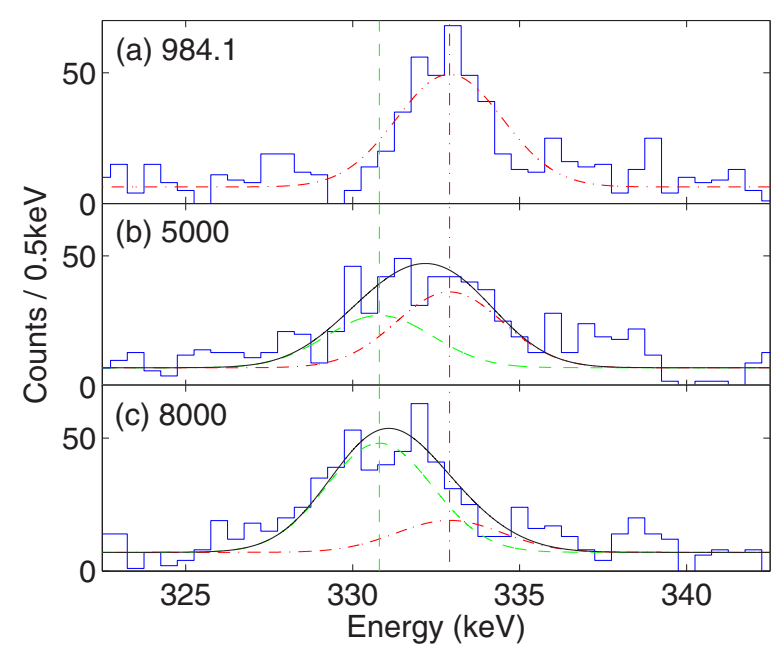

FIG. 2. Examples of recoil-gated $\gamma-\gamma$ coincidence spectra at three different target-to-degrader distances for the $331 \mathrm{keV} \gamma$-ray transition in ${ }^{166} \mathrm{Re}$. The spectra were obtained by the gate set on the $212 \mathrm{keV}$ $\gamma$-ray transition. The dashed green and dot-dashed red vertical lines indicate the fully shifted and degraded components, respectively. Gaussian fits for the two components (dashed and dot-dashed lines), as well as the sum of the two fits (solid line) are also shown in the figure. The shoulder on the right side of the peak at $338 \mathrm{keV}$ is due to the $\gamma$ ray deexciting the $\left(15^{-}\right)$state. The fully shifted components are Doppler corrected with $v / c=4.4 \%$.

the lifetime measurement of the $\left(8^{-}\right)$state. For these two $\gamma$-ray transitions, the degraded components were mostly populated at distances shorter than $984.1 \mu \mathrm{m}$, while both components could be observed at the distances larger than $2000 \mu \mathrm{m}$. Gaussian fits for both the fully-Doppler-shifted and degraded components of the 331 and $398 \mathrm{keV} \gamma$-ray transitions are shown in Figs. 2 and 3 as dashed (green) and dot-dashed

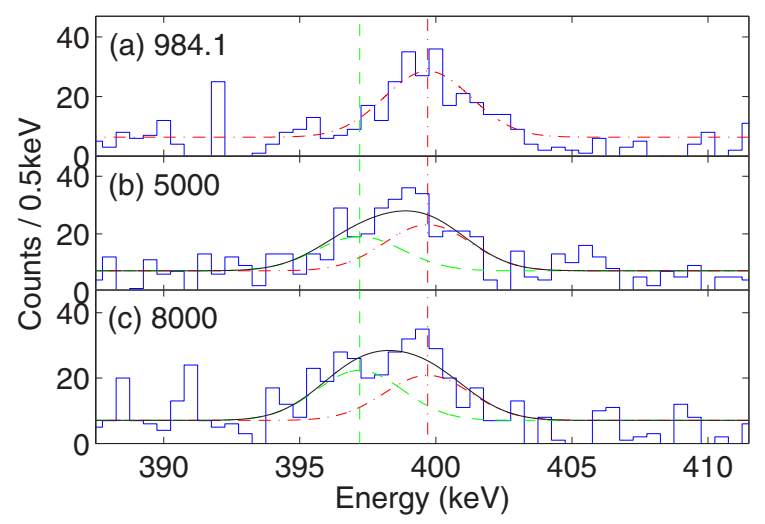

FIG. 3. Examples of recoil-gated $\gamma-\gamma$ coincidence spectra at three different target-to-degrader distances for the $398 \mathrm{keV} \gamma$-ray transition in ${ }^{166} \mathrm{Re}$. The spectra were obtained by the gate set on the $212 \mathrm{keV}$ $\gamma$-ray transition. The dashed green and dot-dashed red vertical lines indicate the fully shifted and degraded components, respectively. Gaussian fits for the two components (dashed and dot-dashed lines), as well as the sum of the two fits (solid line) are also shown in the figure. The fully shifted components are Doppler corrected with $v / c=4.4 \%$.

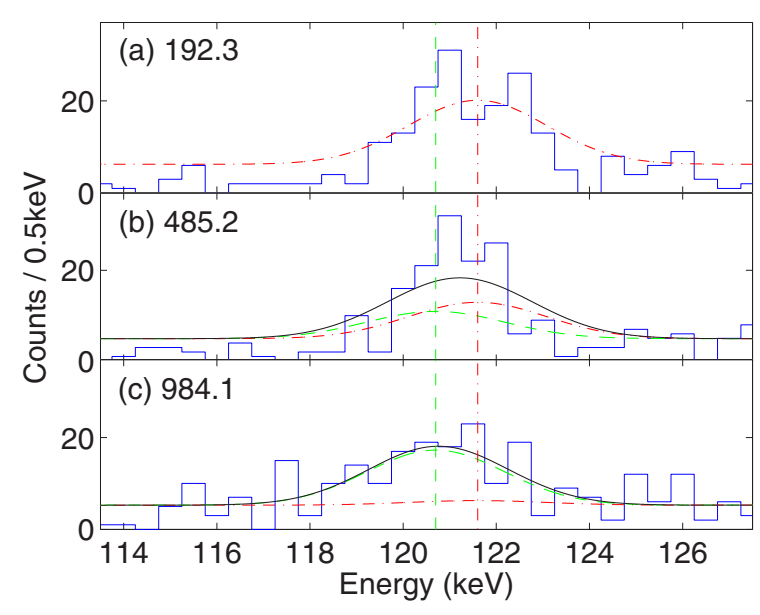

FIG. 4. Examples of recoil-gated $\gamma-\gamma$ coincidence spectra at three different target-to-degrader distances for the $121 \mathrm{keV} \gamma$-ray transition in ${ }^{166} \mathrm{Re}$. The spectra were obtained by the gate on the $225 \mathrm{keV} \gamma$-ray transition. The dashed green and dot-dashed red vertical lines indicate the fully shifted and degraded components, respectively. Gaussian fits for the two components (dashed and dot-dashed lines), as well as the sum of the two fits (solid line) are also shown in the figure. The fully-Doppler-shifted components are Doppler corrected by using $v / c=4.4 \%$.

(red) curves, respectively. The spectra shown in these two figures were obtained by the gate set on both the fully shifted and degraded components of the higher-lying $212 \mathrm{keV} \gamma$-ray transition at target-to-degrader distances of 984.1, 5000, and $8000 \mu \mathrm{m}$. The solid black lines correspond to the sums of the two components. The dashed green and dot-dashed red vertical lines represent the centroids of the fully shifted and degraded components, respectively. The fully-Doppler-shifted $E_{s}$ and degraded $E_{d}$ components for the $\gamma$-ray transitions of interest, the measured lifetime values, together with the energy differences $\Delta E$ between these two parts are shown in Table I. Here the fully shifted components $E_{s}$ agree with the energy values given in Ref. [9]. Figure 4 shows the spectra obtained by the gate set on both the fully shifted and degraded components of the higher-lying $225 \mathrm{keV} \gamma$-ray transition at distances of 192.3, 485.2, and $984.1 \mu \mathrm{m}$. For the $\left(10^{-}\right)$state (see Fig. 5), the spectra were obtained by the gate on both the fully shifted and degraded components of the $301 \mathrm{keV} \gamma$-ray transition at the two shortest distances, i.e., 7.6 and $96.4 \mu \mathrm{m}$. Because the shifted components are not fully separated from the degraded components, peak positions and widths were fixed when fitting the spectra.

The lifetime of the $\left(8^{-}\right)$state can be evaluated by using either the $331 \mathrm{keV} \gamma$-ray transition or the $398 \mathrm{keV} \gamma$ ray transition, taking into account their branching ratios. Figure 6(a) shows the weighted lifetime value obtained from the $331 \mathrm{keV} \gamma$-ray transition, $\tau=440$ (90) ps, within the sensitive region of target-to-degrader distances. The normalized shifted-component intensity [see Fig. 6(b)] was fit with two second-order polynomials. The normalization factors were given by the total counts in the gated spectra of the matrices at the different distances. The deduced lifetime value for the $\left(8^{-}\right)$state from the $398 \mathrm{keV} \gamma$-ray transition, 
TABLE I. Calculation of the energy separation $\Delta E$ between the fully-Doppler-shifted and the degraded components in ${ }^{166} \mathrm{Re}$ at $133.6^{\circ}$. The relative velocities, $v / c$, before and after the degrader were 0.044 and 0.034 , respectively. $E_{s}$ and $E_{d}$ are the values used to fit the Doppler-corrected spectra shown in Figs. 2 to 5.

\begin{tabular}{lllcrrr}
\hline \hline$E_{\gamma}(\mathrm{keV})$ & $E_{i}(\mathrm{keV})$ & $E_{f}(\mathrm{keV})$ & $I_{i}^{\pi} \rightarrow I_{f}^{\pi}$ & $E_{s}(\mathrm{keV})$ & $E_{d}(\mathrm{keV})$ & $\Delta E(\mathrm{keV})$ \\
\hline 121 & $\mathrm{X}+573$ & $\mathrm{X}+452$ & $\left(9^{-}\right) \rightarrow\left(8^{-}\right)$ & 120.7 & 121.6 & 0.9 \\
212 & $\mathrm{X}+785$ & $\mathrm{X}+573$ & $\left(10^{-}\right) \rightarrow\left(9^{-}\right)$ & 211.4 & 212.9 & 1.5 \\
225 & $\mathrm{X}+1010$ & $\mathrm{X}+785$ & $\left(11^{-}\right) \rightarrow\left(10^{-}\right)$ & 224.9 & 226.5 & 1.6 \\
331 & $\mathrm{X}+452$ & $\mathrm{X}+121$ & $\left(8^{-}\right) \rightarrow \mathrm{Z}$ & 330.8 & 332.9 & 2.1 \\
398 & $\mathrm{X}+452$ & $\mathrm{X}+54$ & $\left(8^{-}\right) \rightarrow \mathrm{Y}$ & 397.2 & 399.7 & $440(90)$ \\
\hline \hline
\end{tabular}

$\tau=590$ (150) ps as shown in Fig. 7(a), is consistent within uncertainties with the result obtained from the $331 \mathrm{keV} \gamma$-ray transition. The corresponding normalized shifted component for the $398 \mathrm{keV} \gamma$-ray transition with two second-order polynomials is given in Fig. 7(b). The resulting weighted mean lifetime obtained from both depopulating transitions is $\tau_{\left(8^{-}\right)}=480(80)$ ps.

Figure 8 shows the lifetime analysis for the $\left(9^{-}\right)$state using the $121 \mathrm{keV} \gamma$-ray transition, and the resulting average lifetime value, $\tau=60$ (20) ps. For the $\left(10^{-}\right)$and $\left(11^{-}\right)$states, only upper lifetime limits could be deduced as $\tau_{\left(10^{-}\right)}<1$ ps and $\tau_{\left(11^{-}\right)}<3$ ps, respectively.

Reduced transition probabilities can be deduced for the lowest-order multipolarities from lifetime measurements by using the following relationships:

$$
\begin{aligned}
& B(E 1)=\frac{1}{\tau(1+\alpha) 1.59 \times 10^{15} E_{\gamma}^{3}}\left[e^{2} \mathrm{fm}^{2}\right], \\
& B(E 2)=\frac{1}{\tau(1+\alpha) 1.22 \times 10^{9} E_{\gamma}^{5}}\left[e^{2} \mathrm{fm}^{4}\right],
\end{aligned}
$$

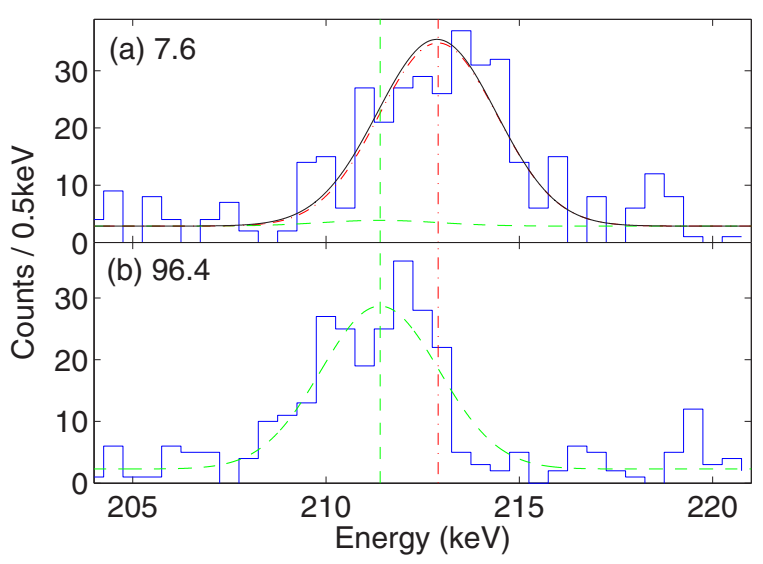

FIG. 5. Examples of recoil-gated $\gamma-\gamma$ coincidence spectra at two different target-to-degrader distances for the $212 \mathrm{keV} \gamma$-ray transition in ${ }^{166} \mathrm{Re}$. The spectra were obtained by the gate on the $301 \mathrm{keV} \gamma$-ray transition. The dashed green and dot-dashed red vertical lines indicate the fully shifted and degraded components, respectively. Gaussian fits for the two components (dashed and dot-dashed lines), as well as the sum of the two fits (solid line) are also shown in the figure. The fully-Doppler-shifted components are Doppler corrected with $v / c=4.4 \%$.

$$
B(M 1)=\frac{1}{\tau(1+\alpha) 1.76 \times 10^{13} E_{\gamma}^{3}}\left[\mu_{N}^{2}\right],
$$

where the lifetime $\tau$ is given in s, the $\gamma$-ray energy $E_{\gamma}$ is given in $\mathrm{MeV}$, and $\alpha$ is the internal conversion coefficient that is here taken from Ref. [30]. The reduced transition probabilities were deduced by assuming pure stretched transitions with no mixing from higher-order multipolarities in formulas (2)-(4). The deduced transition probabilities for the 331 and $398 \mathrm{keV} \gamma$-ray transitions are shown in Table II for different multipolarities taking into account the "missing" intensity of unobserved transitions depopulating the $\left(8^{-}\right)$state. The reduced transition strengths for the 331 and $398 \mathrm{keV} \gamma$-ray transitions are quite similar. It is not very likely that they are $M 1$ transitions since the $B(M 1)$ values are then of the order of $10^{-4}$ W.u., which is about three orders of magnitude weaker than the $M 1$ transitions in the yrast rotational band (see Table II) obtained in the present work. If they are $E 2$ transitions, the reduced transition probabilities are around 1 W.u., which is in agreement with what is expected for a single-particle $E 2$ transition and about 100 times weaker than the collective $E 2$ transitions in the neighboring even-even nuclei [31]. If they are $E 1$ transitions, the $B(E 1)$ values are of the order of $10^{-6}$ W.u., which fall within the systematics [32] for $E 1,|\Delta K| \leqslant 2$ transitions. For example, in the rhenium isotope ${ }^{176} \mathrm{Re}$, the $E 1$ transition, with $B(E 1,99.5 \mathrm{keV})=5.3(5) \times 10^{-6}$ W.u., has been described as a transition from the neutron $i_{13 / 2}[633] 7 / 2^{+}$Nilsson orbital to the neutron $h_{9 / 2}[512] 5 / 2^{-}$Nilsson orbital [33]. Here, one possibility (out of many) could be an $E 1$ transition

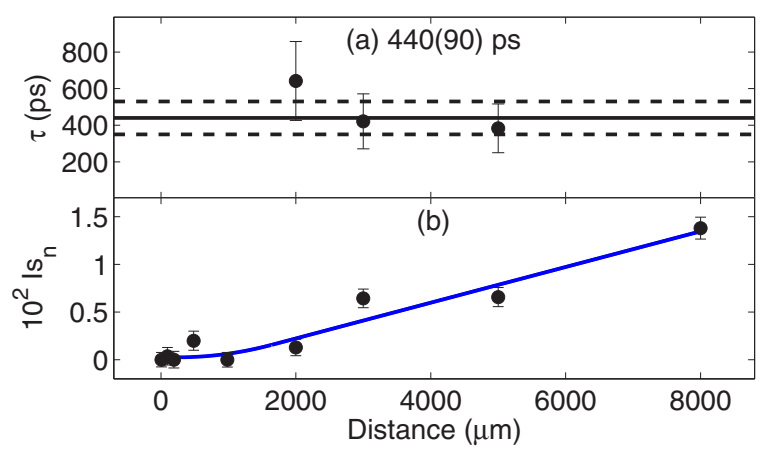

FIG. 6. (a) Deduced lifetime as a function of target-to-degrader distance for the $331 \mathrm{keV} \gamma$-ray transition in ${ }^{166} \mathrm{Re}$. (b) The normalized intensity of the shifted component of the $331 \mathrm{keV} \gamma$-ray transition together with the polynomial fit. 


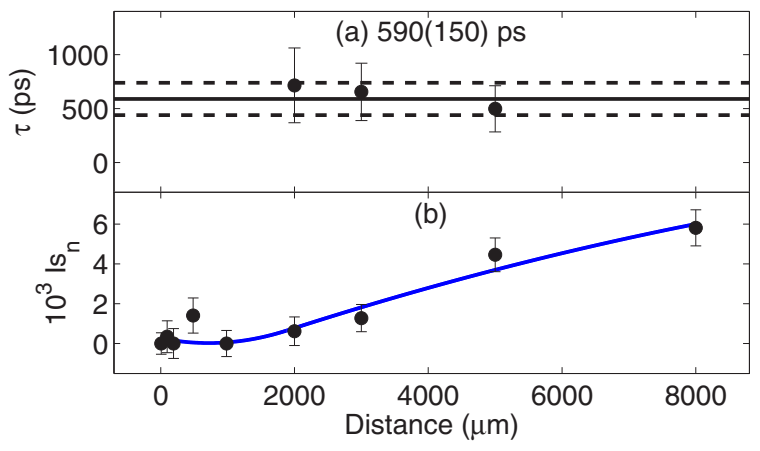

FIG. 7. (a) Deduced lifetime as a function of target-to-degrader distance for the $398 \mathrm{keV} \gamma$-ray transition in ${ }^{166} \mathrm{Re}$. (b) The normalized intensity of the shifted component of the $398 \mathrm{keV} \gamma$-ray transition together with the polynomial fit.

from the proton $h_{11 / 2}[514] 9 / 2^{-}$Nilsson orbital to the proton $g_{7 / 2}[404] 7 / 2^{+}$Nilsson orbital which might result in the spin and parity of the final state being $\left(7^{+}\right)$. We conclude that the 331 and $398 \mathrm{keV}$ transitions are likely to be of either $E 1$ or $E 2$ character. Hence, there are numerous possibilities for the spin-parities of the two states populated by these transitions in the range $I^{\pi}=6^{-}, 7^{+}$to $I^{\pi}=9^{+}, 10^{-}$.

\section{DISCUSSION}

\section{A. Collective strength in the yrast band of ${ }^{166} \mathrm{Re}$}

Rotational-like band structures in ${ }^{166} \mathrm{Re}$ identified for the first time by Li et al. [9] revealed two strongly coupled rotational structures. The intense $\gamma$-ray cascade was assigned to the yrast $\pi h_{11 / 2}[514] 9 / 2^{-} \otimes v i_{13 / 2}[660] 1 / 2^{+}$Nilsson configuration while the less strongly populated structure was tentatively assigned to be built on a two-quasiparticle state of $\pi h_{11 / 2} \otimes$ $v h_{9 / 2} / f_{7 / 2}$ character. The configuration assignments were based on the electromagnetic characteristics and rotational patterns; e.g., quasiparticle alignment and signature splitting, in comparison with the predictions from total Routhian surface (TRS) and particle-rotor model calculations [9]. The TRS calculations predict the yrast configuration to be based on a nearaxially symmetric deformation with $\beta_{2}=0.17$; see Fig. 9 . The ratios of electromagnetic transition rates $B(M 1) / B(E 2)$ were also interpreted within the semiclassical Dönau and Frauendorf approach [34] by using the results from the TRS calculations for the predictions of the $E 2$ transition rates.

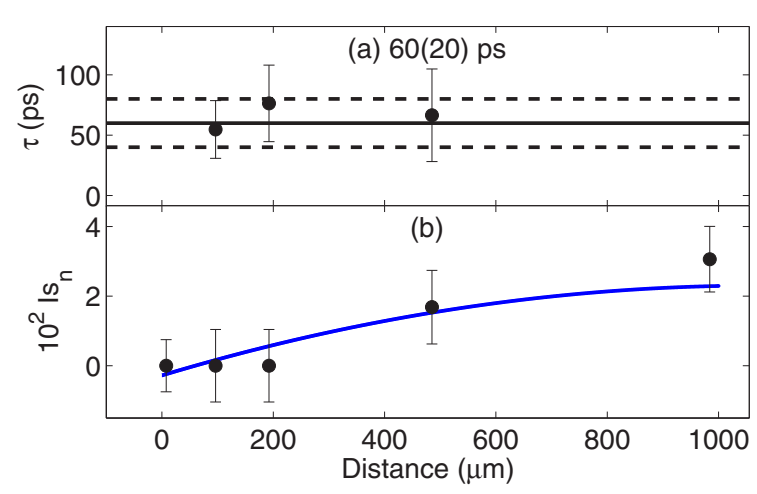

FIG. 8. (a) Deduced lifetime as a function of target-to-degrader distance for the $121 \mathrm{keV} \gamma$-ray transition in ${ }^{166} \mathrm{Re}$. (b) The normalized intensity of the shifted component of the $121 \mathrm{keV} \gamma$-ray transition together with the polynomial fit.

Reduced transition probabilities are important probes of nuclear structure. The measurements of absolute electromagnetic transition rates for excited states in the yrast band structure performed in this work hence provide further opportunities to test the model predictions. We also extend the theoretical investigations to tilted axis cranking relativistic mean-field theory (TAC-RMF) [35] in order to assess the possibility of magnetic rotation as an alternative scenario for explaining the observed band structures (see below).

Within the semiclassical collective model, the $M 1$ transition strengths are given by [34]

$$
\begin{aligned}
B(M 1, I \rightarrow I-1) & =|\langle I I|\mu(M 1)| I-1 I-1\rangle|^{2} \\
& =\left(3 \mu_{\perp}^{2}\right) /(8 \pi),
\end{aligned}
$$

where $\mu_{\perp}$ is the transverse magnetic moment and can be expressed as

$$
\begin{aligned}
\mu_{\perp}= & \left(g_{K p}-g_{R}\right)\left[\Omega_{p}\left(1-K^{2} / I^{2}\right)^{1 / 2}-i_{p} K / I\right] \\
& +\left(g_{K n}-g_{R}\right)\left[\Omega_{n}\left(1-K^{2} / I^{2}\right)^{1 / 2}-i_{n} K / I\right] .
\end{aligned}
$$

Here, $g_{K p}$ and $i_{p}$ are the $g$ factor and alignment for the deformation-aligned valence proton, $g_{K n}$ and $i_{n}$ are the $g$ factor and alignment for the valence neutron and the collective $g$ factor $g_{R}$ is taken as $Z / A$. The single-particle $g$ factors were taken from Ref. [36] and the single-particle alignments

\begin{tabular}{|c|c|c|c|c|c|c|}
\hline Energy $(\mathrm{keV})$ & $B(M 1) \downarrow\left(\mu_{N}^{2}\right)$ & $B(M 1) \downarrow$ (W.u.) & $B(E 2) \downarrow\left(e^{2} \mathrm{fm}^{4}\right)$ & $B(E 2) \downarrow$ (W.u.) & $B(E 1) \downarrow\left(\times 10^{-5} e^{2} \mathrm{fm}^{2}\right)$ & $B(E 1) \downarrow\left(\times 10^{-6}\right.$ W.u. $)$ \\
\hline 331 & $3.7_{-0.5}^{+0.7} \times 10^{-4}$ & $2.0_{-0.2}^{+0.2} \times 10^{-4}$ & $53_{-7}^{+10}$ & $1.0_{-0.1}^{+0.2}$ & $0.6_{-0.1}^{+0.1}$ & $2.4_{-0.3}^{+0.5}$ \\
\hline 398 & $2.1_{-0.3}^{+0.4} \times 10^{-4}$ & $1.2_{-0.1}^{+0.1} \times 10^{-4}$ & $20_{-3}^{+4}$ & $0.4_{-0.1}^{+0.1}$ & $0.3_{-0.1}^{+0.1}$ & $1.3_{-0.2}^{+0.3}$ \\
\hline 121 & $0.10_{-0.02}^{+0.04}$ & $0.06_{-0.01}^{+0.01}$ & & & & \\
\hline 212 & $>3.0$ & $>1.7$ & & & & \\
\hline 225 & $>0.9$ & $>0.5$ & & & & \\
\hline 333 & & & $>6525$ & $>120$ & & \\
\hline 437 & & & $>13555$ & $>250$ & & \\
\hline
\end{tabular}
were taken from Ref. [9]. The total angular momentum projected on the nuclear symmetry axis, $K=\Omega_{p}+\Omega_{n}$,

TABLE II. The reduced transition probabilities for different $\gamma$-ray transitions in ${ }^{166}$ Re. 


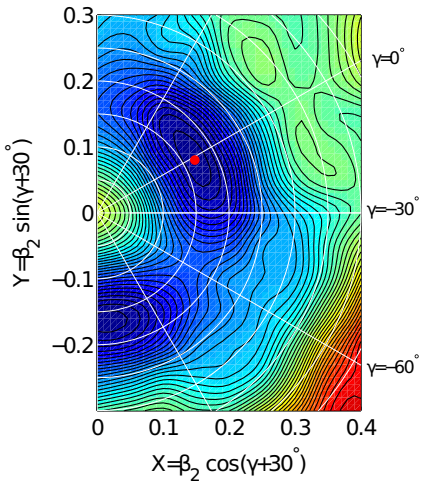

FIG. 9. Total Routhian surface plot for ${ }^{166} \mathrm{Re}$ at $\hbar \omega=0.0$ $\mathrm{MeV}$ taken from the previous work [9]. The minimum at $\left(\beta_{2}, \gamma\right)=\left(0.17,-1.8^{\circ}\right)$ corresponds to the yrast $\pi h_{11 / 2}[514] 9 / 2^{-} \otimes$ $v i_{13 / 2}[660] 1 / 2^{+}$Nilsson configuration. The energy difference between successive contour curves is $200 \mathrm{keV}$.

follows the Gallagher-Moszkowski rule [37]. The resulting prediction for the reduced transition probability $B(M 1)$ for the $121 \mathrm{keV} \gamma$-ray transition is $0.10 \mu_{N}^{2}$. The experimental limits $\geqslant 3.0 \mu_{N}^{2}$ for the $212 \mathrm{keV} \gamma$-ray transition and $\geqslant 0.9 \mu_{N}^{2}$ for the $225 \mathrm{keV} \gamma$-ray transition are in agreement with the predictions from the semiclassical collective model, while the value of around $0.10 \mu_{N}^{2}$ for the $\left(9^{-}\right) \rightarrow\left(8^{-}\right)$transition is much smaller; see Table II. This may point to a significant mixing with other single-particle configurations in the $\left(8^{-}\right)$ band head. Using double-coincidence gates on the (398, 225), $(398,301)$, and $(398,296) \mathrm{keV}$ transition pairs, the branching ratio $I(E 2 ; 333 \mathrm{keV}) / I(M 1 ; 212 \mathrm{keV})$ of the $\left(10^{-}\right)$ state is measured to be $0.036(10)$. The limit of the reduced electric quadrupole transition probability can then be obtained as $B\left(E 2 ;\left(10^{-}\right) \rightarrow\left(8^{-}\right)\right)>6525 e^{2} \mathrm{fm}^{4}$ and $B\left(E 2 ;\left(11^{-}\right) \rightarrow\right.$ $\left.\left(9^{-}\right)\right)>13555 e^{2} \mathrm{fm}^{4}$. The deformation parameter $\beta_{2}$ can be deduced from the quadrupole moment, within the framework of the rigid-rotor model, by using the formula [38]

$$
Q_{0}=\frac{3}{\sqrt{5 \pi}} R^{2} Z \beta_{2}\left(1+0.16 \beta_{2}\right),
$$

with $R=R_{0} A^{1 / 3}$ and $R_{0}=1.4 \mathrm{fm}$. Using the relationship

$$
B(E 2 ; I \rightarrow I-2)=\frac{5}{16 \pi} Q_{0}^{2}\langle I K 20 \mid I-2 K\rangle^{2},
$$

a $\beta_{2}$ value of 0.177 for $\left(10^{-}\right)$and 0.235 for $\left(11^{-}\right)$is obtained, similar to the values obtained for the nuclei ${ }^{168} \mathrm{~W}$ and ${ }^{172} \mathrm{Os}$ with $\beta_{2} \sim 0.23$ [31]. However, it is somewhat higher than the TRS prediction of $\beta_{2}=0.17$.

\section{B. Possible magnetic rotation in ${ }^{166}$ Re: analysis based on tilted axis cranking relativistic mean-field theory}

The "shears mechanism" [39] and magnetic rotation [40], which are observed as rotational-like patterns of excitation energies in some weakly deformed or near-spherical nuclei, has received much attention in the last decades. Experimentally, more than 200 magnetic rotational bands spread over 90 nuclides have been identified so far. Most of them are reported in the mass regions $A \sim 60, A \sim 80, A \sim 110, A \sim 130$,

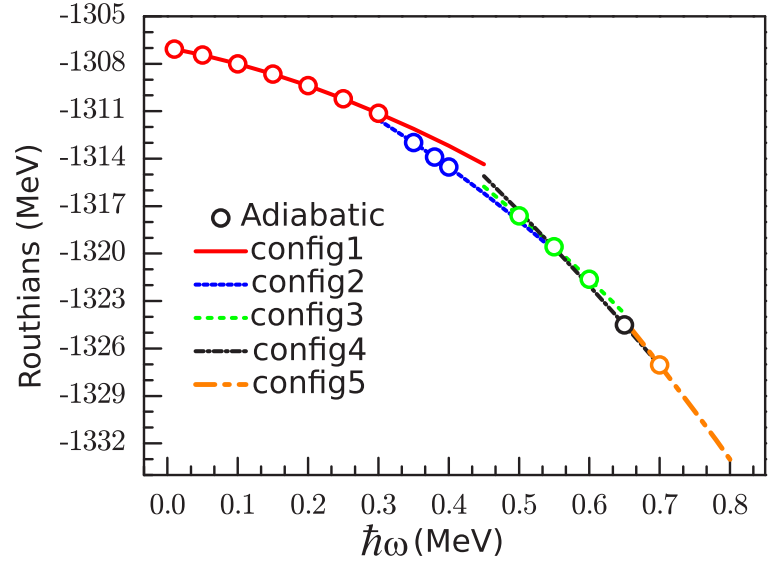

FIG. 10. The total Routhians as functions of the rotational frequency for ${ }^{166} \mathrm{Re}$ in adiabatic (open circles) and configuration-fixed (lines) TAC-RMF calculations with effective interaction PC-PK1.

and $A \sim 190$ [41-44]. The nucleus ${ }^{166}$ Re fulfills the basic conditions of a magnetic rotational structure. The valence proton may occupy a deformation-aligned orbital, while the valence neutron may favor a rotation-aligned configuration. Hence, the total angular momentum in excited states can, in principle, be generated by means of the shears mechanism where excited states are produced by successive alignments of the initially perpendicular single-particle angular momentum vectors without the influence of a collective rotational motion.

Relativistic mean-field (RMF) theory [45-47] has received wide attention due to its success in describing properties of nuclei and many nuclear phenomena in the past few decades. Based on the RMF theory, the tilted axis cranking relativistic mean-field theory has been developed for describing the nuclear magnetic and antimagnetic rotational modes [44]. The cranking RMF model with arbitrary orientation of the rotational axis, i.e., three-dimensional cranking, has been developed in Ref. [35]. Because of its numerical complexity, it has so far been applied only for the magnetic rotation in ${ }^{84} \mathrm{Rb}$ [35]. Focusing on this phenomenon, the two-dimensional cranking RMF theory based on the meson exchange [48] and the point-coupling interactions $[49,50]$ were established. They considerably simplify the numerical complexity and have been applied successfully to describe magnetic rotation in ${ }^{22} \mathrm{~F},{ }^{58} \mathrm{Fe}$, ${ }^{60} \mathrm{Ni},{ }^{113} \mathrm{In},{ }^{114} \mathrm{In},{ }^{142} \mathrm{Gd},{ }^{198,199} \mathrm{~Pb}$, etc. [44].

When investigating the spectroscopic properties of the ${ }^{166} \mathrm{Re}$ nucleus in this work, the point-coupling interaction PC-PK1 [51] was used and pairing correlations were neglected. A spherical harmonic oscillator basis with 10 major shells was employed to solve the Dirac equation. Possible configurations in ${ }^{166} \mathrm{Re}$ were searched in the following steps: (i) Adiabatic TAC-RMF calculations $[48,52]$ were performed to search for the possible configurations. Here the term "adiabatic" means that the nucleons always occupy the lowest singleparticle levels during the constraint process. This leads to the results shown as open circles in Fig. 10. By analyzing the occupation of the valence nucleons and the corresponding transformed spherical quantum numbers (for details, see Ref. [48]), five possible configurations were obtained. 
TABLE III. The deformation parameters $\beta_{2}$ and $\gamma$, and their corresponding configurations (valence nucleon and unpaired nucleon) as well as the parities for configurations config1-5 in the TAC-RMF calculations.

\begin{tabular}{|c|c|c|c|c|}
\hline Notation & $\left(\beta_{2}, \gamma\right)$ & Valence nucleon configuration & Unpaired nucleon configuration & $\pi$ \\
\hline config2 & $\left(0.23,13.1^{\circ}\right)$ & $\pi\left[h_{11 / 2}^{-4} h_{9 / 2}^{1} d_{3 / 2}^{2}\right] \otimes v\left[i_{13 / 2}^{2} h_{9 / 2}^{-5} f_{7 / 2}^{2}\right]$ & $\pi h_{9 / 2}^{1} \otimes v\left[i_{13 / 2}^{2} h_{9 / 2}^{-1} f_{7 / 2}^{2}\right]$ & + \\
\hline config3 & $\left(0.21,17.4^{\circ}\right)$ & $\pi\left[h_{11 / 2}^{-4} h_{9 / 2}^{1} d_{3 / 2}^{2}\right] \otimes v\left[i_{13 / 2}^{2} h_{9 / 2}^{-5} f_{7 / 2}^{2}\right]$ & $\pi\left[h_{11 / 2}^{-2} h_{9 / 2}^{1}\right] \otimes v\left[i_{13 / 2}^{2} h_{9 / 2}^{-1} f_{7 / 2}^{2}\right]$ & + \\
\hline config4 & $\left(0.28,26.3^{\circ}\right)$ & $\pi\left[i_{13 / 2}^{1} h_{11 / 2}^{-4} h_{9 / 2}^{1} d_{3 / 2}^{1}\right] \otimes v\left[i_{13 / 2}^{3} h_{9 / 2}^{4} f_{7 / 2}^{3} d_{3 / 2}^{-1}\right]$ & $\pi\left[i_{13 / 2}^{1} h_{9 / 2}^{1} d_{3 / 2}^{1}\right] \otimes v\left[i_{13 / 2}^{3} f_{7 / 2}^{3} d_{3 / 2}^{-1}\right]$ & + \\
\hline
\end{tabular}

(ii) Starting from the different points corresponding to the five configurations (different-colored open circles in Fig. 10), TACRMF calculations with the configuration-fixed constraint were performed. Here the term "configuration-fixed" means that the nucleons occupy the same combination of single-particle levels with increasing rotational frequency. The results for each configuration are shown as different lines in Fig. 10 with different colors corresponding to the different configurations. The detailed configuration information is listed in Table III.

In Table III, the five valence nucleons and unpaired nucleon configurations with their deformation parameters and parities are listed. The values of $\beta_{2}$ and $\gamma$ correspond to the deformation parameters obtained at the lowest rotational frequency for a given configuration. For example, the deformation parameters $\left(\beta_{2}, \gamma\right)$ are $\left(0.21,15.0^{\circ}\right)$ at $\hbar \omega=0.05 \mathrm{MeV}$ for the most energetically favored configuration (config1). In addition, note that the valence nucleon configuration is different from the unpaired nucleon configuration. Taking config 1 as an example, for the valence proton, there are three holes in the $h_{11 / 2}$ orbital and two particles occupying the $d_{3 / 2}$ orbital. Therefore, the valence proton configuration is written as $\pi\left[h_{11 / 2}^{-3} d_{3 / 2}^{2}\right]$. However, two $h_{11 / 2}$ holes and two $d_{3 / 2}$ particles are paired, respectively. Thus the unpaired proton configuration is written as $\pi h_{11 / 2}^{-1}$. Similarly, for the valence neutron, there is one particle in the $i_{13 / 2}$ orbital, four holes in the $h_{9 / 2}$ orbital, and two particles in the $f_{7 / 2}$ orbital. Out of these, the four $h_{9 / 2}$ holes and two $f_{7 / 2}$ particles are paired. Therefore, the valence neutron and unpaired nucleon configuration are written as $v\left[i_{13 / 2}^{1} h_{9 / 2}^{-4} f_{7 / 2}^{2}\right]$ and $\nu i_{13 / 2}^{1}$, respectively. It is noted that the two $f_{7 / 2}$ particles will soon become unpaired with increasing rotational frequency.

The total angular momenta as a function of the rotational frequency in adiabatic (open circles) and configuration-fixed (lines) constrained TAC-RMF calculations are plotted in comparison with the data of bands (1) and (2) of ${ }^{166} \operatorname{Re}[9]$ in Fig. 11. The experimental rotational frequency is deduced as [53]

$$
\hbar \omega_{\text {expt }}=\frac{1}{2}\left[E_{\gamma}(I+1 \rightarrow I)+E_{\gamma}(I \rightarrow I-1)\right] .
$$

All of the calculated angular momenta $I(\omega)$ for each configuration form nearly straight lines. The calculated results based on config1 are closest to the experimental data for band (1), although it can be seen that the TAC-RMF results overestimate the experimental values. This may result from neglecting the pairing correlations in the present TAC-RMF calculations, which enhances the alignment contributions from the two neutrons in the $f_{7 / 2}$ orbital which are closest to the Fermi level.

A key characteristic of magnetic rotation is strongly enhanced $M 1$ transitions between states differing by one unit of angular momentum at low angular momenta as well as their decreasing tendency with increasing angular momentum. Correspondingly, there are weak $\Delta I=2 E 2$ transitions in such bands. In Fig. 12, the electromagnetic transition probability ratios $B(M 1) / B(E 2)$ [9], as well as individual $B(M 1)$ and $B(E 2)$ values from the TAC-RMF calculations with the most favored configuration, config1, for band (1), are shown as a function of the total angular momentum in comparison with the semiclassical collective model predictions. The experimental ratios $B(M 1) / B(E 2)$ take into account internal conversion (which was not the case for the values given in Ref. [9]). Similarly to Ref. [49], the $B(M 1)$ values obtained from the TACRMF model are attenuated by a factor 0.3 . With the exception of $B\left(M 1 ;\left(10^{-}\right) \rightarrow\left(9^{-}\right)\right) / B\left(E 2 ;\left(10^{-}\right) \rightarrow\left(8^{-}\right)\right)$where the experimental uncertainty is large, the $B(M 1) / B(E 2)$ ratios obtained from the TAC-RMF calculations overshoot the experimental values while the semiclassical model gives a fair description of the experimental data. The calculated $B(M 1)$ values using the collective model are also consistent with the experimental $B(M 1)$ limits obtained for the 212 and $225 \mathrm{keV} \gamma$-ray transitions. On the other hand, the experimental

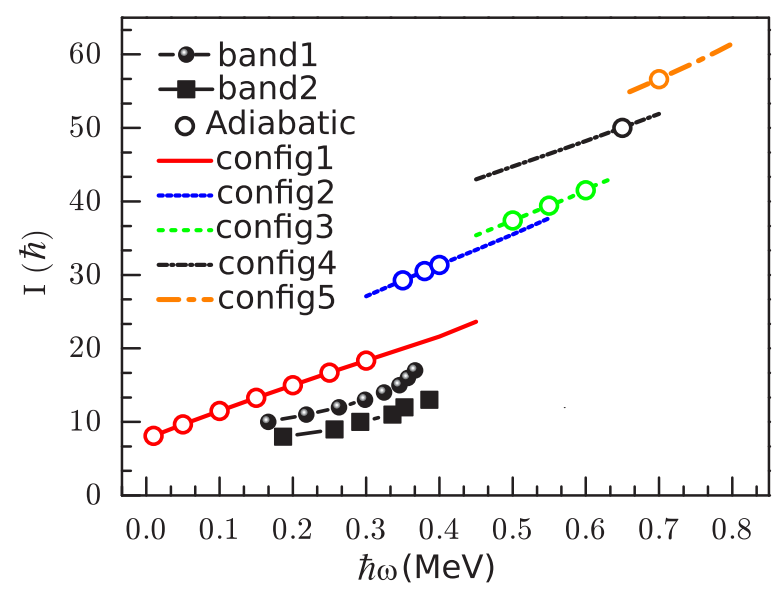

FIG. 11. Total angular momenta as a function of the rotational frequency in adiabatic (open circles) and configuration-fixed (lines) constrained TAC-RMF calculations with effective interaction PCPK1 compared with the data of bands (1) and (2) in ${ }^{166} \mathrm{Re}$. 


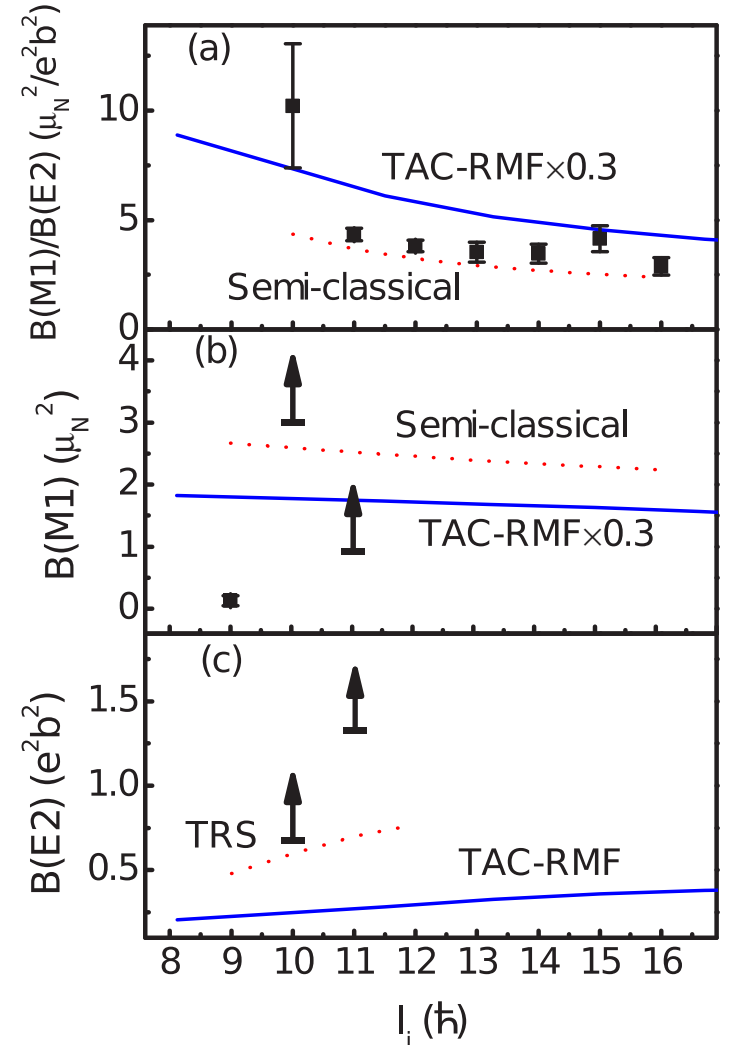

FIG. 12. Experimental information (given as data points and lower limits) on (a) $B(M 1) / B(E 2)$ ratios, (b) $B(M 1)$ values, and (c) $B(E 2)$ values as a function of the total angular momentum (tentative assignment) compared with theoretical calculations (see text) for band (1) in ${ }^{166} \mathrm{Re}$. Note that here the experimental $B(M 1) / B(E 2)$ ratios have been evaluated by taking into account internal conversion (assuming pure multipolarity) and hence differ somewhat from those given in Ref. [9].

$B(M 1)$ value from the $\left(9^{-}\right)$state is much lower, indicating that there might be strong configuration mixing close to the band head. The $B(E 2)$ value deduced from the TRS calculation is relatively close to the experimental $B(E 2)$ limit from the same state. Turning to the TAC-RMF calculations, while the tendency of calculated $B(M 1)$ and $B(E 2)$ values are consistent with the characteristics of magnetic rotation, the agreement with the experimental data is worse than for the collective model. Figure 13 shows the predicted deformation parameters $\beta_{2}$ and $\gamma$ obtained from the TAC-RMF calculations for the five lowest configurations in ${ }^{166} \mathrm{Re}$ (config1-5). However, there is a preference for nonaxial shapes in the TAC-RMF predictions. It can also be seen in Fig. 13 that the deformation parameter $\beta_{2}$ for the five configurations becomes slightly smaller while $\gamma$ becomes larger with increasing rotational frequency. While the TAC-RMF calculations for config1 predict a quadrupole deformation only slightly different from the result of the TRS calculations, the predicted $B(E 2)$ values are smaller. This illustrates the importance of experimentally determined electromagnetic transition rates for constraining nuclear structure theory. In this case the present lifetime measurements of excited states in the yrast band of ${ }^{166} \mathrm{Re}$ provide a means to distinguish between TAC-RMF predictions

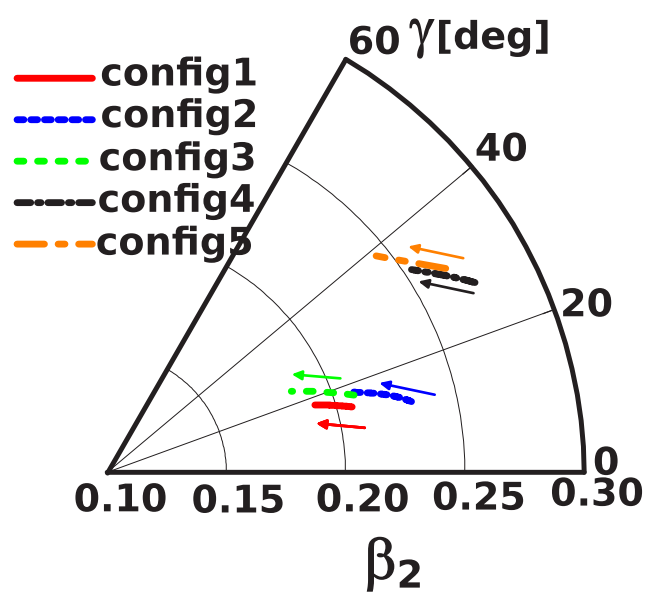

FIG. 13. Evolutions of deformation parameters $\beta_{2}$ and $\gamma$ driven by increasing rotational frequency in the TAC-RMF calculations for config1-5 for ${ }^{166} \mathrm{Re}$. The arrows indicate the increasing direction of rotational frequency.

of magnetic rotation and the predictions from the collective rotational model. The present investigation suggests that band (1) of ${ }^{166} \mathrm{Re}$ is not an ideal example of magnetic rotation but is better characterized as a collective rotational system.

\section{SUMMARY}

Lifetime measurements have been performed for the three excited states in the yrast band of ${ }^{166}$ Re by using the RDDS method. The data were collected with the DPUNS plunger device located at the target position of the JUROGAM II array at the Accelerator Laboratory of the University of Jyväskylä, Finland. The deduced $B(E 2)$ limit from the $\left(10^{-}\right)$state is reasonably consistent with the prediction from TRS calculations and the systematics. Both the deduced $B(M 1) / B(E 2) *$ and $B(M 1)$ values for this state agree with the predictions from collective-rotational-model calculations for the $\pi h_{11 / 2}[514] 9 / 2^{-} \otimes v i_{13 / 2}[660] 1 / 2^{+} \quad$ Nilsson configuration while the experimental $B(M 1)$ value obtained for the $\left(9^{-}\right) \rightarrow\left(8^{-}\right)$transition is much lower and indicates mixing with other single-particle configurations in the bandhead configuration. The observed yrast band (1) in ${ }^{166} \mathrm{Re}$ has also been studied in the framework of TAC-RMF theory. The calculated total angular momenta with configuration $\pi h_{11 / 2}^{-1} \otimes$ $v i_{13 / 2}^{1}$ as a function of the rotational frequency overestimates the experimental values of band (1). This may result from the contribution of two aligned particles in the $f_{7 / 2}$ orbital. The predicted strong $M 1$ transition rates as well as very weak $E 2$ transitions for configuration $\pi h_{11 / 2}^{-1} \otimes v i_{13 / 2}^{1}$ are characteristic of magnetic rotation. However, the TAC-RMF predictions for magnetic rotation in ${ }^{166} \mathrm{Re}$ differ from the experimental data, which follow rather well with the semiclassical calculations as well as TRS predictions. This suggests that band (1) of ${ }^{166} \mathrm{Re}$ is not an ideal example of magnetic rotation, but has significant contributions from collective rotation. This work highlights the importance of measurements of absolute electromagnetic transitions strengths for stringent tests of nuclear structure theory. 


\section{ACKNOWLEDGMENTS}

The authors would like to thank the staff at the Accelerator Laboratory of the University of Jyväskylä for their excellent technical support. This work was supported by the Swedish Research Council (Project No. 621-2010-3694), the Academy of Finland under the Finnish Centre of Excellence Programme 2012 - 2017, the UK Science and Technology Facilities
Council, and the European Union Seventh Framework Programme Integrating Activities-Transnational Access, Project No. 262010 (ENSAR). Li is supported in part by the China Scholarship Council under Grant No. 201306210205. Additionally, we thank the GAMMAPOOL European Spectroscopy Resource for the loan of the detectors for JUROGAM II array. T.G. acknowledges the support from the Academy of Finland (Contract No. 131665).
[1] P. Moller, R. Bengtsson, B. G. Carlsson, P. Olivius, and T. Ichikawa, Phys. Rev. Lett. 97, 162502 (2006).

[2] A. J. Kreiner and M. A. J. Mariscotti, Phys. Rev. Lett. 43, 1150 (1979).

[3] A. J. Kreiner and M. A. J. Mariscotti, J. Phys. G: Nucl. Phys. 6, L13 (1980).

[4] C. X. Yang and H. Y. Zhou, Chin. Phys. Lett. 20, 2140 (2003).

[5] L. L. Liedinger et al., Acta Phys. Pol. B 32, 2613 (2001).

[6] Y. Liu, Y. Ma, H. Yang, and S. Zhou, Phys. Rev. C 52, 2514 (1995).

[7] T. Komatsubara et al., Nucl. Phys. A 557, 419 (1993).

[8] Y. H. Zhang, Nucl. Phys. A 834, 32c (2010).

[9] H. J. Li, M. Doncel, M. Patial, B. Cederwall, T. Back, U. Jakobsson, K. Auranen, S. Bonig, M. Drummond, T. Grahn, P. Greenlees, A. Herzan, D. T. Joss, R. Julin, S. Juutinen, J. Konki, T. Kroll, M. Leino, C. McPeake, D. O’Donnell, R. D. Page, J. Pakarinen, J. Partanen, P. Peura, P. Rahkila, P. Ruotsalainen, M. Sandzelius, J. Saren, B. Saygi, C. Scholey, J. Sorri, S. Stolze, M. J. Taylor, A. Thornthwaite, J. Uusitalo, and Z. G. Xiao, Phys. Rev. C 92, 014310 (2015).

[10] R. Bengsston et al., Nucl. Phys. A 415, 189 (1984).

[11] I. Hamamoto, Phys. Lett. B 235, 221 (1990).

[12] K. Hara and Y. Sun, Nucl. Phys. A 531, 221 (1991).

[13] M. J. Taylor et al., Nucl. Instrum. Methods Phys. Res., Sect. A 707, 143 (2013).

[14] C. W. Beausang et al., Nucl. Instrum. Methods Phys. Res., Sect. A 313, 37 (1992).

[15] C. Rossi Alvarez, Nucl. Phys. News 3, 10 (1993).

[16] G. Duchêne, F. A. Beck, P. J. Twin, G. de France, D. Curien, L. Han, C. W. Beausang, M. A. Bentley, P. J. Nolan, and J. Simpson, Nucl. Instrum. Methods Phys. Res., Sect. A 432, 90 (1999).

[17] M. Leino et al., Nucl. Instrum. Methods Phys. Res., Sect. B 99, 653 (1995).

[18] M. Leino, Nucl. Instrum. Methods Phys. Res., Sect. B 126, 320 (1997).

[19] J. Sarén, J. Uusitalo, M. Leino, and J. Sorri, Nucl. Instrum. Methods Phys. Res., Sect. A 654, 508 (2011).

[20] R. D. Page et al., Nucl. Instrum. Methods Phys. Res., Sect. B 204, 634 (2003).

[21] I. H. Lazarus et al., IEEE Trans. Nucl. Sci. 48, 567 (2001).

[22] P. Rahkila, Nucl. Instrum. Methods Phys. Res., Sect. A 595, 637 (2008).

[23] D. C. Radford, Nucl. Instrum. Methods Phys. Res., Sect. A 361, 297 (1995).

[24] D. B. Fossan and E. K. Warburton, Pure and Applied Physics, edited by J. Cerny, Vol. 40-C (Academic Press, New York, 1974), p. 307.
[25] T. K. Alexander and J. S. Forster, Adv. Nucl. Phys. 74, 197 (1978).

[26] A. Dewald, O. Möller, and P. Petkov, Prog. Part. Nucl. Phys. 67, 786 (2012).

[27] A. Dewald, S. Harissopulos, and P. von Brentano, Z. Phys. A 334, 163 (1989).

[28] G. Böhm, A. Dewald, P. Petkov, and P. von Brentano, Nucl. Instrum. Methods Phys. Res., Sect. A 329, 248 (1993).

[29] F. Seiffert, Program APATHIE, Institut für Kernphysik, Universitat zu Köln, 1989 (unpublished).

[30] T. Kibédi, T. W. Burrows, M. B. Trzhaskovskaya, P. M. Davidson, and C. W. Nestor Jr., Nucl. Instrum. Methods Phys. Res., Sect. A 589, 202 (2008).

[31] S. Raman, C. W. Nestor Jr., and P. Tikkanen, At. Data Nucl. Data Tables 78, 1 (2001).

[32] K. E. G. Löbner, in The Electromagnetic Interaction in Nuclear Spectroscopy, edited by W. D. Hamilton (North-Holland, Amsterdam, 1975), p. 141.

[33] M. A. Cardona, A. J. Kreiner, D. Hojman, G. Levinton, M. E. Debray, M. Davidson, J. Davidson, R. Pirchio, H. Somacal, D. R. Napoli, D. Bazzacco, N. Blasi, R. Burch, D. DeAcuna, S. M. Lenzi, G. LoBianco, J. Rico, and C. R. Alvarez, Phys. Rev. C 59, 1298 (1999).

[34] F. Dönau and S. Frauendorf, in Proceedings of the Conference on High Angular Momentum Properties of Nuclei, Oak Ridge, edited by N. R. Johnson (Harwood, New York, 1982), p. 143.

[35] H. Madokoro, J. Meng, M. Matsuzaki, and S. Yamaji, Phys. Rev. C 62, 061301 (2000).

[36] S. Frauendorf, Phys. Lett. B 100, 219 (1981).

[37] C. J. Gallagher, and S. A. Moszkowski, Phys. Rev. 111, 1282 (1958).

[38] K. S. Krane, in Introductory Nuclear Physics (John Wiley \& Sons, New York, 1988), p.142.

[39] G. Baldsiefen et al., Nucl. Phys. A 574, 521 (1994).

[40] S. Frauendorf, J. Meng, and J. Reif, in Proceedings of the Conference on Physics from Large $\gamma$-Ray Detector Arrays, Vol. II of Report LBL35687, edited by M. A. Deleplanque (Univ. of California, Berkeley, 1994), p. 52.

[41] Amita, Ashok, K. Jain, and B. Singh, At. Data Nucl. Data Tables 74, 283 (2000).

[42] S. Frauendorf, Rev. Mod. Phys. 73, 463 (2001).

[43] H. Hübel, Prog. Part. Nucl. Phys. 54, 1 (2005).

[44] J. Meng, J. Peng, S. Q. Zhang, and P. W. Zhao, Front. Phys. 8, 55 (2013).

[45] P. Ring, Prog. Part. Nucl. Phys. 37, 193 (1996).

[46] D. Vretenar, A. V. Afanasjev, G. A. Lalazissis, and P. Ring, Phys. Rep. 409, 101 (2005).

[47] J. Meng, H. Toki, S. Zhou, S. Zhang, W. Long, and L. Geng, Prog. Part. Nucl. Phys. 57, 470 (2006). 
[48] J. Peng, H. Sagawa, S. Q. Zhang, J. M. Yao, Y. Zhang, and J. Meng, Phys. Rev. C 77, 024309 (2008).

[49] P. W. Zhao, S. Q. Zhang, J. Peng, H. Z. Liang, P. Ring, and J. Meng, Phys. Lett. B 699, 181 (2011).

[50] P. W. Zhao, J. Peng, H. Z. Liang, P. Ring, and J. Meng, Phys. Rev. C 85, 054310 (2012).
[51] P. W. Zhao, Z. P. Li, J. M. Yao, and J. Meng, Phys. Rev. C 82, 054319 (2010).

[52] J. Meng, J. Peng, S. Q. Zhang, and S. G. Zhou, Phys. Rev. C 73, 037303 (2006).

[53] S. Frauendorf and J. Meng, Z. Phys. A: Hadrons Nucl. 356, 263 (1996). 